Politik in Nordamerika und Europa 
Jan Grasnick • Katja Walter (Hrsg.)

\section{Politik in Nordamerika und Europa}

Analysen, Theorien

und literarische Rezeption

Springer VS 
Herausgeber

Jan Grasnick,

Katja Walter,

Augsburg, Deutschland

ISBN 978-3-531-19497-4

ISBN 978-3-531-19498-1 (eBook)

DOI 10.1007/978-3-531-19498-1

Die Deutsche Nationalbibliothek verzeichnet diese Publikation in der Deutschen Nationalbibliografie; detaillierte bibliografische Daten sind im Internet über http://dnb.d-nb.de abrufbar.

Springer VS

(C) VS Verlag für Sozialwissenschaften | Springer Fachmedien Wiesbaden 2012

Das Werk einschließlich aller seiner Teile ist urheberrechtlich geschützt. Jede Verwertung, die nicht ausdrücklich vom Urheberrechtsgesetz zugelassen ist, bedarf der vorherigen $\mathrm{Zu}-$ stimmung des Verlags. Das gilt insbesondere für Vervielfältigungen, Bearbeitungen, Übersetzungen, Mikroverfilmungen und die Einspeicherung und Verarbeitung in elektronischen Systemen.

Die Wiedergabe von Gebrauchsnamen, Handelsnamen, Warenbezeichnungen usw. in diesem Werk berechtigt auch ohne besondere Kennzeichnung nicht zu der Annahme, dass solche Namen im Sinne der Warenzeichen- und Markenschutz-Gesetzgebung als frei zu betrachten wären und daher von jedermann benutzt werden dürften.

Einbandentwurf: KünkelLopka GmbH, Heidelberg

Gedruckt auf säurefreiem und chlorfrei gebleichtem Papier

Springer VS ist eine Marke von Springer DE. Springer DE ist Teil der Fachverlagsgruppe Springer Science+Business Media

www.springer-vs.de 


\section{Vorwort}

\section{Einleitung}

Das Jahr 2011 wird für Rainer-Olaf Schultze immer ein besonderes sein. Es ist das Jahr seines Eintritts in den „Ruhestand“. An den man jedoch nicht ganz glauben mag, da bekanntlich mit 66 Jahren das Leben ja erst anfängt. Wie auch immer Rainer-Olaf Schultze seinen Ruhestand gestaltet, wir freuen uns darüber, ihn vor diesen neuen Abschnitt seines Lebens für das Zurückliegende zu würdigen. Wir, das sind seine Kollegen und Schüler, Wegbegleiter und Freunde.

So heterogen diese Gruppe auch sein mag, alle Beteiligten sind durch Wissenschaft, Forschung und Lehre mit Rainer-Olaf Schultze verbunden und teilen mehr oder weniger bestimmte Gegenstandsbereiche, mit denen sich der zu Ehrende im Laufe seiner langen wissenschaftlichen Karriere auseinandergesetzt hat. So ist ein Buch entstanden, das sich unter den vielfältigen Perspektiven verschiedener Disziplinen der Politik und den Gesellschaften Nordamerikas und Europas widmet. Aus literatur- und sprachwissenschaftlicher Sicht befassen sich Martin Kuester, Katja Sarkowsky und Lothar Wolf mit der kanadischen Gesellschaft und ihrer historischen Entwicklung. Eine politikwissenschaftliche Perspektive nehmen Tim Nieguth, Ralf Lindner, Falko Brede und Jörg Broschek ein, während sich Günther Kronenbitter mit dem Krisenmanagement des Nachbarn im Süden in der Kuba-Krise aus Sicht der Geschichtswissenschaft auseinandersetzt. Den Fokus auf Europa und die Europäische Union richten Jan Grasnick, der regionales Regieren und Demokratie in der EU untersucht, Katharina Holzinger mit einer Abhandlung über Probleme kollektiven Handelns und HansOtto Mühleisen mit der Frage danach, wie viel Vertrauen bzw. Misstrauen eine Demokratie braucht; Dieter Nohlen setzt sich mit der politischen Kultur Spaniens und Deutschlands auseinander und Katja Walter betrachtet vergleichend idealtypische Krisenphänomene und die Reaktionsmuster der Politik darauf. Allen Beiträgern gilt unser herzlicher Dank.

Es ist nicht leicht, die Forschungsgebiete von Rainer-Olaf Schultze auf einen Nenner zu bringen. Gesellschaftliche Konflikte und politische Intervention in den Staaten der ,atlantischen“ Welt - so könnte man sein Forschen und Lehren stark verkürzt umschreiben. Eine andere, vielleicht treffendere Formulierung die auch den Wandel der Politik und ihrer Rolle aufzeigt - wäre „Politische Integration und Regieren in Nordamerika und Europa“. Konkret befasste er sich in

seiner wissenschaftlichen Tätigkeit hauptsächlich mit Vergleichender Politik und 
Politischer Soziologie industrieller Demokratien mit den Area-Schwerpunkten Nordamerika (Kanada, USA) und Westeuropa (Großbritannien, Bundesrepublik Deutschland), Vergleichender Föderalismus-Forschung (unter Einschluss Kanadas, der USA, der Bundesrepublik Deutschland und Australiens), Empirischer Wahlforschung sowie Verfassungspolitik und Politikberatung. Die immer wieder zu beobachtende Fokussierung auf Nordamerika und Europa soll auch der Strukturierung dieser Festschrift dienen und damit verdeutlichen, dass sich die verwandten Gesellschaften diesseits und jenseits des Atlantiks mitunter erheblich voneinander unterscheiden können.

\section{Heidelberger und Bochumer Jahre}

In seiner Heimatstadt Heidelberg nahm Rainer-Olaf Schultze 1965 an der Ruprecht-Karls Universität das Studium der Politischen Wissenschaft, Geschichte und Romanistik auf, das er dort auch zehn Jahre später mit der Promotion zum Dr. phil. abschloss. Bereits in den ersten Studiensemestern kam Schultze intensiv mit der Forschung in Berührung. Er trat dem Forschungsseminar „Europäisches Wahlrecht" bei, das nominell von Dr. Bernhard Vogel und faktisch bereits von Dieter Nohlen geleitet wurde, seit Vogel 1965 in den Bundestag gewählt worden war. Dieser Umstand eröffnete dem jungen Forscher Schultze die Chance, Vogel bei Recherche und Niederschrift des Beitrags „Deutschland“ zum Handbuch „Die Wahl der Parlamente“ (herausgegebenen von Dolf Sternberger und Bernhard Vogel, Redaktion Dieter Nohlen) beizustehen und diesen am Ende in KoAutorschaft mit dem späteren Ministerpräsidenten von Rheinland-Pfalz und Thüringen zu verantworten.

Im Rahmen des Forschungsseminars entwickelte sich auch die vielfältige, thematisch breiter angelegte Zusammenarbeit mit Dieter Nohlen. Sie umfasste gemeinsam verfasste Aufsätze und Herausgeberschaften zu Wahlen und Wählerverhalten, regionaler Entwicklung und Politikwissenschaft als wissenschaftlicher Disziplin. Am bekanntesten ist wohl das „Lexikon der Politikwissenschaft“, das über die Jahre inzwischen die vierte Auflage (2010) erlebte. Nohlen erinnert sich lebhaft an Ko-Autorschaften mit Schultze: Man traf sich in Nohlens Büro, formulierte frei Gedanken zum jeweiligen Gegenstand, präzisierte sie im Dialog, setzte sie auf und feilte anschließend an den Formulierungen. Das so entstandene vorläufige Manuskript ging nachher jeder für sich noch einmal auf theoretische Schlüssigkeit und empirische Tragfähigkeit durch. In den für notwendig erachteten Korrekturen stimmte man sich dann meistens telefonisch ab. In dieser Art der Zusammenarbeit verwirklichten sich einige der großen Vorzüge Schultzes: seine stete Bereitschaft, in einem ernsten, kreativen, im Prinzip uneigennützigen, wenn 
angesagt an konkreten Ergebnissen interessierten Dialog einzutreten. Viele Studienfreunde und Kollegen haben so auch von seiner enormen Belesenheit profitiert.

Als 1969 das Forschungsprojekt „Außerparlamentarisches Wahlrecht“ von der DFG bewilligt wurde, dessen Leitung Dieter Nohlen und Klaus Landfried übernahmen, hatte dies wegweisende Bedeutung für Schultzes wissenschaftlichen Werdegang. Zum einen blieb er der Wahlforschung erhalten und lieferte sich bald in Sachen bundesdeutscher Wahlentwicklung spannende Gefechte mit dem Mainstream der deutschen Wahlforschung. Er hat praktisch zu jeder Bundestagswahl eine Wahlanalyse verfasst, von denen die meisten in der ,Zeitschrift für Parlamentsfragen" veröffentlicht wurden. Zum anderen übernahm er in dem neuen Projekt, an dem auch Franz Nuscheler und Klaus Ziemer beteiligt waren, den Länderbeitrag Kanada. Es ergab sich dann geradezu zwangsläufig, Kanada auch zum Gegenstand der Dissertation zu wählen. Hier liegen also die Wurzeln der Kanada-Orientierung Schultzes. Später nahm er noch - angeregt durch den Dialog mit Walter D. Burnham (,,the leading expert on elections and voting patterns in the United States") und begünstigt durch ein Studienjahr an der Harvard University - die USA als Forschungsgegenstand hinzu. Diesen Forschungsinteressen konnte Schultze verstärkt an der Ruhr-Universität in Bochum nachgehen, als er dort 1976 als wissenschaftlicher Assistent von Erwin Faul angestellt wurde; eine Tätigkeit, die er bis 1985 ausübte. Dort machte er sich einen Namen als Nordamerikaforscher, zumal mit der Veröffentlichung seiner außergewöhnlich umfangreichen Dissertation „Politik und Gesellschaft in Kanada“, die zu Publikationszwecken auf „magere 620 Seiten“ gekürzt werden musste.

\section{Kanada im Blickpunkt: \\ Rainer-Olaf Schultze an der Universität Augsburg}

Anknüpfend an die Zeit in Heidelberg und Bochum führte Rainer-Olaf Schultze nach seinem Ruf an die Universität Augsburg seine wissenschaftliche Tätigkeit besonders zu Kanada fort. Glücklicherweise als nicht beispielhaft für seinen forscherischen Weitblick und seine Beziehung zu Kanada zeigte sich sein Versuch, im Jahr 1997 die Tagung der Canadian Political Science Association (CPSA) an der Memorial University in St. John's, Neufundland, zu besuchen. Während er dort von seinen Mitarbeitern bereits dringend erwartet wurde, öffnete sich stattdessen die Tür im Augsburger Institut für Kanada-Studien und Professor Schultze spazierte herein. Aufgrund überaus dichten Nebels war keine Landung in St. John's möglich gewesen, weshalb das Flugzeug die Militärbasis Stephenville anflog. Die Aussicht auf eine Nacht auf der spartanisch ausgestatte- 
ten Airbase, einen zehnstündigen Bustransfer nach St. John's am nächsten Tag und möglicherweise eine Woche ohne Gepäck, das in Stephenville nicht ausgeladen werden konnte, veranlassten ihn dazu, alles in Bewegung zu setzen, um seinem Koffer nicht von der Seite zu weichen. Trotz anfänglicher Weigerung des Flugpersonals, Rainer-Olaf Schultze mit zurück nach Europa zu nehmen, konnte er sich schließlich durchsetzen und hatte damit seinen wohl kürzesten Aufenthalt auf kanadischem Boden hinter sich gebracht.

Auch wenn Schultze in diesem Fall seine ganze Energie dafür einsetzte, Kanada so schnell wie möglich wieder zu verlassen, zeigt die Anekdote doch, dass er ein „Nein“ nicht unhinterfragt akzeptiert. Dies durften auch seine Kolleginnen und Kollegen im universitären Betrieb vor allem während seiner langjährigen Gremientätigkeit, unter anderem als Dekan der Philosophisch-Sozialwissenschaftlichen Fakultät, immer wieder erfahren. Während dieser setzte Professor Schultze sich stets besonders für die Interessen der Politikwissenschaft im Allgemeinen und die der Kanadistik im Besonderen ein.

Der fast schon symbolisch anmutende dichte Nebel, der sich im Frühsommer über Neufundland gelegt hatte und Rainer-Olaf Schultze daran hinderte, an der CPSA-Tagung teilzunehmen, zeigte sich zum Glück nicht symptomatisch für seine Tätigkeit am Augsburger Institut für Kanada-Studien, dem er seit dessen Gründung im Jahr 1985 als geschäftsführender Direktor vorsteht. Das Institut für Kanada-Studien ist eine bundesweit einzigartige interdisziplinäre Einrichtung, an dem neben der Politikwissenschaft auch Romanistik, Anglistik, Geschichte und bis zum Ausscheiden von Prof. Roland Vogelsang - Geographie beteiligt sind. Mit seiner wissenschaftlichen Arbeit an der Universität Augsburg hat Professor Schultze die Kanadistik in Forschung und Lehre ebenso maßgeblich geprägt und ihr zu Ansehen verholfen wie durch seine persönlichen Kontakte zu Vertretern der kanadischen Politik. Durch seine Gründungsmitgliedschaft in der Gesellschaft für Kanadastudien (GKS) und die langjährige Aktivität in dieser wissenschaftlichen Vereinigung war er zudem an der Schaffung eines Austauschforums für die Kanadistik im gesamten deutschsprachigen Raum beteiligt, das wiederum zu neuen Erkenntnissen, Erfahrungen und Perspektiven beitragen konnte. Man kann Professor Schultze also getrost als einen der Pioniere der interdisziplinären Kanada-Forschung in Deutschland bezeichnen.

Das fächerübergreifende Projekt „Kanada-Studien an der Universität Augsburg" hat die exogenen Schocks sowohl des Eintretens Rainer-Olaf Schultzes in den Ruhestand als auch der Bologna-Reform, der die Magisterstudiengänge anheim fielen, gut überstanden und besteht in der Lehre fort als nicht unwesentlicher Bestandteil des Masters „Nordamerikastudien“, der - nicht zuletzt angeregt durch Rainer-Olaf Schultze - von den Mitgliedern des Kanada-Instituts 
gemeinsam entwickelt wurde und den durchaus erfolgreichen Magisterstudiengang „Kanadistik“ ablöst.

\section{Danksagung}

Unser Dank gilt allen, die das Zustandekommen dieser Festschrift möglich gemacht haben. Neben den Beiträgern sind dies vor allem Claudia Glöckner, Rainer-Olaf Schultzes langjährige Mitarbeiterin am Institut, die studentischen Hilfskräfte Sebastian Geßler, Anna Kozaczek, Nicolas Güttler und Jessica Detemple, und die Verantwortlichen des VS-Verlages Dorothee Koch und Sabine Schöller. Unser besonderer Dank gilt zudem Dieter Nohlen, der uns bei der Verfassung dieses Vorwortes unterstützt hat. Nicht zuletzt bedanken wir uns für die finanzielle Unterstützung seitens der Gesellschaft für Kanada-Studien, die zur Verwirklichung dieses Projekts beigetragen hat.

Augsburg, im März 2012

Katja Walter und Jan Grasnick 


\section{Inhalt}

Vorwort

\section{Themengebiet Nordamerika}

Falko Brede

Defizite der kanadischen Innovations- und Forschungspolitik

Ralf Lindner

E-Konsultationen in Kanada -

Vorbild für Deutschland oder Sonderfall

Jörg Broschek

Historical Institutionalism and the Study of Canadian Politics

Martin Kuester

"'Tis Ninety Years Since":

The Great War in British and Canadian Novels

Günther Kronenbitter

Realismus und Vertrauen -

Anmerkungen zum Krisenmanagement der USA 1962

Tim Nieguth

Westphalian Territoriality in Diverse Societies:

Majority Nationalism and the Manitoba School Question

Katja Sarkowsky

Ambivalente Einschreibungen:

,Citizenship' und ,Nation' in japanisch-kanadischer Literatur

Lothar Wolf

Zur Entstehung der kanadischen Varietät des Französischen. 


\section{Themengebiet Europa}

Dieter Nohlen

Politische Kultur in Spanien und Deutschland

Jan Grasnick

Regionales Regieren und Demokratie in der Europäischen Union

Katharina Holzinger

Inequality in Collective Action Problems

Katja Walter

Krisenphänomene und Reaktionsmuster der Politik im Vergleich

Hans-Otto Mühleisen

Vertrauen in der Politik

Verzeichnis der Autorinnen und Autoren 